\title{
RECENT DEVELOPMENTS IN MULTIPLE CRITERIA DECISION MAKING
}

\author{
Stanley Zionts and Vahid Lotfi \\ School of Management \\ State University of New York at Buffalo \\ Buffalo, New York 14260 U. S. $\Lambda$.
}

\section{ABSTRAC'}

Problems involving more than one criterion abound. To help in the solution of such problems, a field of management science and operations research known as multiple criteria decision making ( $M C D M)$ has emerged to help solve such problems. In this paper we discuss some recent developments in this important field.

\section{INTRODUCTION}

The purpose of this paper is to overview multiple criteria decision making (MCDM) and to consider certain recent developments in the field. MCDM refers to making decisions in the presence of multiple, usually conflicting, noncommensurate objectives. Multiple criteria decision problems pervade all that we do and is very general indeed.

In this paper, we will first introduce certain MCDM concepts, then introduce several prototypical examples of MCDM problems, and then discuss the recent developments.

\section{SOME MCDM CONCEPTS}

1. The Concept of Dominance

An important concept in multiple criteria problems is the concept of dominance. To describe this concept, we would like to use an analogy with a town in the state of Pennsylvania called Northeast. The town is so named because it is the most northeast town in the state. More specifically, there is no other part of Pennsylvania north and east of it. Consider the map of Pennsylvania shown in Figure 1. The location of the 
http://orion.journals.ac.za/

2

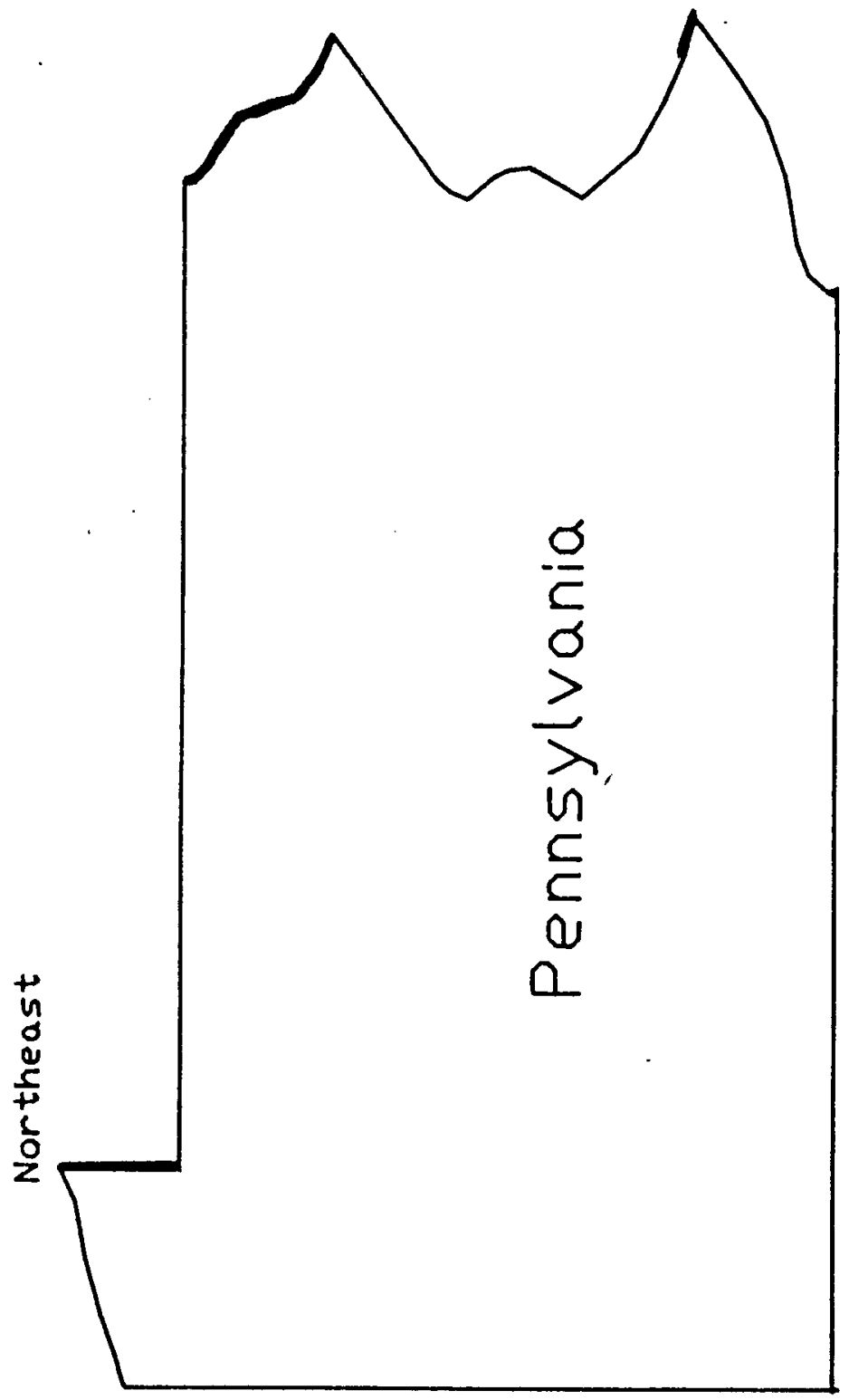

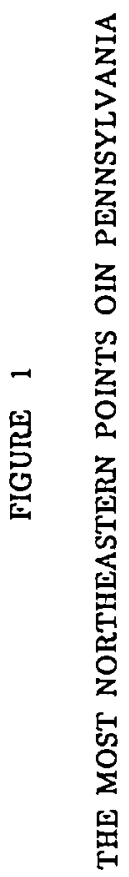


town of Northeast may be a bit of a surprise to many readers not familiar with this town, but as promised there is no part of Pennsylvania to the north or east of it. Are there any other parts of Pennsylvania which share the same characteristics, namely that there are no parts of Pennsylvania to the north and east? Of course there are, and we have indicated them using a bold border.

The concept of having no points to the north and east in a set is closely related to the concept of dominance in MCDM. Consider a decision problem in which there are two objectives, both to be maximized. For example, suppose that a person is choosing between several possible jobs on the basis of salary and job satisfaction, both of which he wishes to maximize. Assuming that we can measure job satisfaction on a scale ranging from 0 to 10 , and the salaries range from $\$ 10,000$ to $\$ 50,000$ per year, we may plot the various possible jobs on a graph as in Figure 2. Except for trivial situations in which an individual can find a job which has both a high salary and high level of job satisfaction, he must make compromises in finding a job. One individual may want a high salary and be willing to settle for low job satisfaction, and another may want high job satisfaction and be willing to settle for a low salary, and so on. Provided that we have no other factors to consider in making this decision, whichever solution we choose should not have any points having at least as great a salary and at least as much job satisfaction. Graphically, there should be no solutions to the northeast of the chosen solution.

Solutions which have no solutions "to their northeast". in this way are said to be nondominated solutions. There are no other solutions which are as good in all objectives and strictly better in at least one objective. Solutions which have solutions to their northeast are said to be dominated solutions, because one of the solutions to the northeast is at least as good in all objectives and strictly better in at least one. In choosing a job from the possibilities, so long as there are no other considerations omitted from our analysis, we should choose one that is nondominated.

The argument is slightly different when some objectives are to be minimized or set at certain levels, but the underlying idea is still the same.

\section{How to Choose a (Nondominated) Solution}

How do we choose a solution from the set of nondominated solutions? First, assume without loss of generality that we are maximizing all of the objectives. (A slight modification must be made if this is not true.) There are four naive methods of selection that we might think of.

First, we might try to choose target levels of all objectives and then find a solution that attains all of these target levels. Unfortunately, we are almost certain to set our targets too high or too low. If we set them too high, there is no solution that 


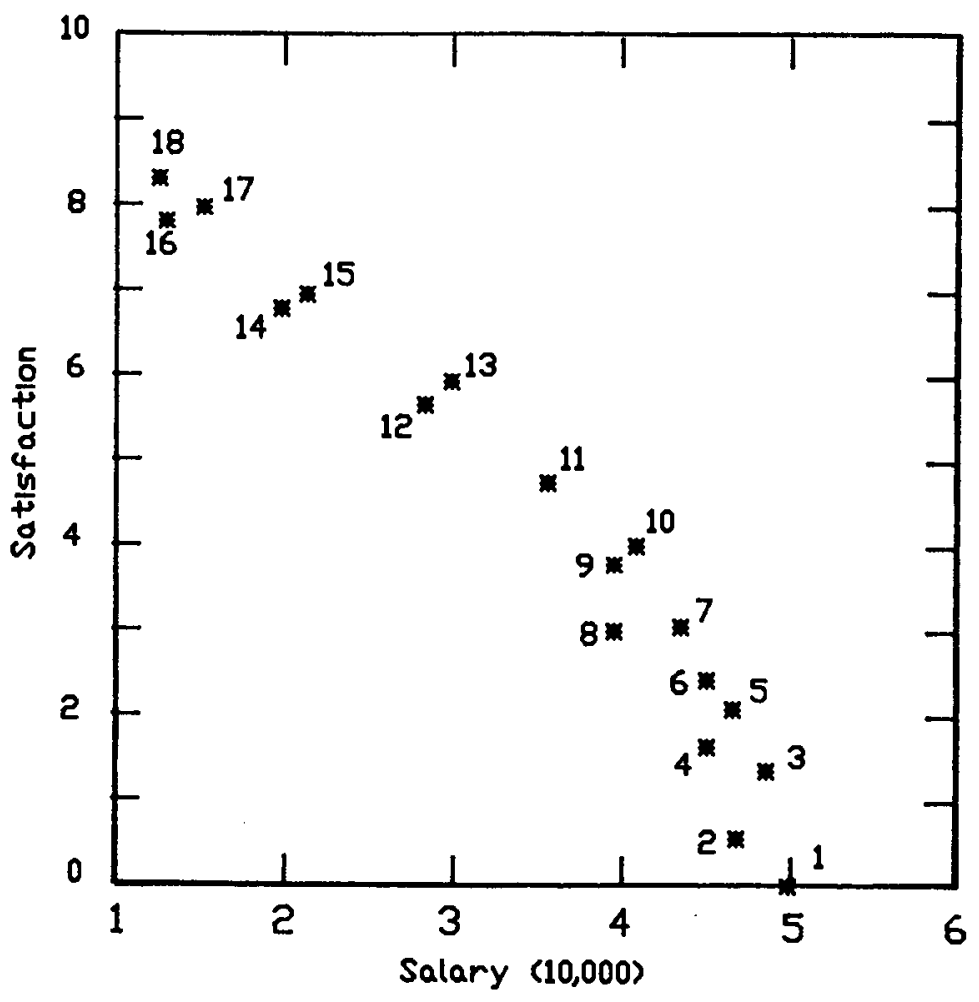

FIGURE 2 
achieves the levels sought, and if we set them too low, a solution that attains those levels is dominated.

Second, we might set minimal levels on all the objectives but one and then find a solution that maximizes the one objective while satisfying the minimal levels of the other objectives. The problem in doing this is that the process does not necessarily yield a nondominated solution.

Third, we might try to identify all nondominated solutions to a problem (i.e., all of the northeast points). Unfortunately, the number of such solutions may be enormous. Identifying all of these solutions is a huge task and then deciding which one of the large number of solutions to choose is also difficult.

Fourth, we may use some mathematical function to combine the objectives into a superobjective. (Such a superobjective, if it exists, is called a value function if the outcomes are known for certain, and a utility function if the outcomes are stochastic.) The problem is what function to use. Suppose we limit ourselves to the choice of a linear function, which means we need only determine a set of positive weights to use in constructing our superobjective. Unfortunately, for a given ranking of alternatives, the weights of a linear function are not unique. Steuer (1986) gives an interesting presentation illustrating how the use of weights in different though similar situations may give rise to erroneous results. Specifically, the weights used to identify a given nondominated solution are generally not unique. As a result, one cannot transfer the weights used for one set of decisions to a similar set of decisions.

Though these methods are naive and have problems associated with them, most methods proposed and used in solving multiple criteria problems use one or more of these naive approaches, but combine them and invoke special procedures to overcome the difficulties that may arise.

Would we ever want to consider alternatives that are dominated? The answer would seem to be an obvious no, so long as 1) we have included all of the criteria in the analysis; and 2) that the criteria fully reflect the problem. Unfortunately, in practice, these conditions may not hold, in spite of the apparent goodness of nondominated solutions. In such situations, we may want to consider choosing a dominated solution to a problem. For example, consider the purchase of a house. An individual may wish to minimize cost, distance to schools, and distance to shopping. However, criteria such as floor plan may not be incorporated in the analysis and may nonetheless be important. Unless we come up with a way of categorizing different floor plans and evaluating them, that criterion may have to be treated only on an ad hoc basis. The well-defined criteria will be used to choose which houses to look at, and the less well-defined criteria will be used in making the decision. In addition, for the second point above (the criteria fully reflecting the problem), the cost criterion that we 
use in our analysis is the asking price, as opposed to transaction price, which is the relevant price. The transaction price is usually a bit lower than the asking price, perhaps about ten percent. This factor varies among houses, and is a result of negotiation. Consequently, a house with a higher asking price than another may. ultimately have a lower transaction price. So, in spite of the attractiveness of nondominated solutions in principle, we should not blindly exclude dominated solutions from consideration.

\section{SOME PROTOTYPICAL EXAMPLES}

In this section we present some prototypical examples of multiple criteria decision problems. We present three kinds of examples in all, each being an example of a different type of problem.

\section{Discrete Alternative Problems}

The first kind of problem is a problem which has a discrete number of alternatives. First, we consider the problem illustrated in Figure 2. A person looking for a job has a number of alternatives. The criteria important to him are salary and job satisfaction, both of which are to be maximized. Of the solutions in Figure 2, solutions 2,4,8,9,12,14 and 16 are dominated and should not be considered further, so long as there are no factors other than salary and job satisfaction that are relevant to the decision. This problem is easier than one involving more than two objectives. Having removed any dominated solutions, we need only search along one dimension, e.g., salary, to determine a mostpreferred position because there is a direct tradeoff from one objective (salary) to the other (job satisfaction). As one objective increases, the other decreases. For example, if we were working in terms of decreasing salary, we would proceed with the nondominated solutions in the order $1,3,5,6,7,10,11,13$, $15,17,18$.

To illustrate a problem involving more than two objectives, consider the purchase of a microcomputer, which an individual or a company wants to purchase. There are a large number of alternative computers, about 150 in all. The criteria identified by the buyer includes price, primary storage capacity, secondary capacity, speed and quality of the monitor. All of the criteria are to be maximized, except for price which is to be minimized. Because of the number of objectives, it is not possible to treat this example graphically as we did in the job-selection problem. (There is a graphical representation that we could use if there were only three objectives. We will briefly discuss that representation in the next section.) We can, however, visualize this problem as selecting a row from a matrix where each of the rows is an alternative and each of the columns give the score for each alternative on one of the objectives. 


\section{Multiple Objective Linear Programuning Problerns}

Unlike the discrete alternative problem, the multiple objective linear programming problem generally has infinitely many solutions. These solutions come about because the constraints on the problem are written mathematically, and the solutions are expressed in real numbers, and are not required to be integers. For such problems, there are usually infinitely many nondominated solutions as well.

Consider the following problem: A company has four products that it can produce: products one, two, three, and four. There are two different production facilities that are used to process each of the products. There are three objectives, each of which is to be maximized. The complete statement of the problem is as follows:

$$
\begin{aligned}
\operatorname{Maximize} \mathrm{u}_{1}= & 3 \mathrm{x}_{1}+\mathrm{x}_{2}+2 \mathrm{x}_{3}+\mathrm{x}_{4} \\
\mathrm{u}_{2}= & \mathrm{x}_{1}-\mathrm{x}_{2}+2 \mathrm{x}_{3}+4 \mathrm{x}_{4} \\
\mathrm{u}_{3}= & -\mathrm{x}_{1}+5 \mathrm{x}_{2}+\mathrm{x}_{3}+2 \mathrm{x}_{4} \\
\text { Subject to: } \quad & 2 \mathrm{x}_{1}+\mathrm{x}_{2}+4 \mathrm{x}_{3}+3 \mathrm{x}_{4}<=60 \quad\left(\text { slack } \mathrm{x}_{5}\right) \\
& 3 \mathrm{x}_{1}+4 \mathrm{x}_{2}+\mathrm{x}_{3}+2 \mathrm{x}_{4}<=60 \quad\left(\text { slack } \mathrm{x}_{6}\right) \\
& \mathrm{x}_{1}, \mathrm{x}_{2}, \mathrm{x}_{3}, \mathrm{x}_{4}>=0
\end{aligned}
$$

Unlike simple two variable problems, which can be solved by graphically plotting in two dimensions, this problem cannot be plotted graphically. A two-dimensional projection of the nondominated solutions for this problem may be plotted (see Figure 3). There is no convenient way in which to search the surface to find a most preferred solution for a three (or more) objective problem. A very convenient way of exploring the two-dimensional projection of a three-objective problem is developed in a computer program by Climaco and Antunes (1987) in an approach called Trimap.

\section{Bargaining or Multiple Decision-maker Problems}

An important class of multiple criteria decision making problems is those that involve more than one party. Such problems are known as bargaining or multiple decision-maker problems.

Consider the following problem: Labor and management are negotiating over four issues to be agreed upon in a labor contract: 1. Wage rate; 2. Number of weeks of vacation; 3. Job security level; and 4. Level of benefits. In order that their negotiations be resolved, both sides must agree unanimously on a solution. How should they resolve their problem and come to an agreement? 


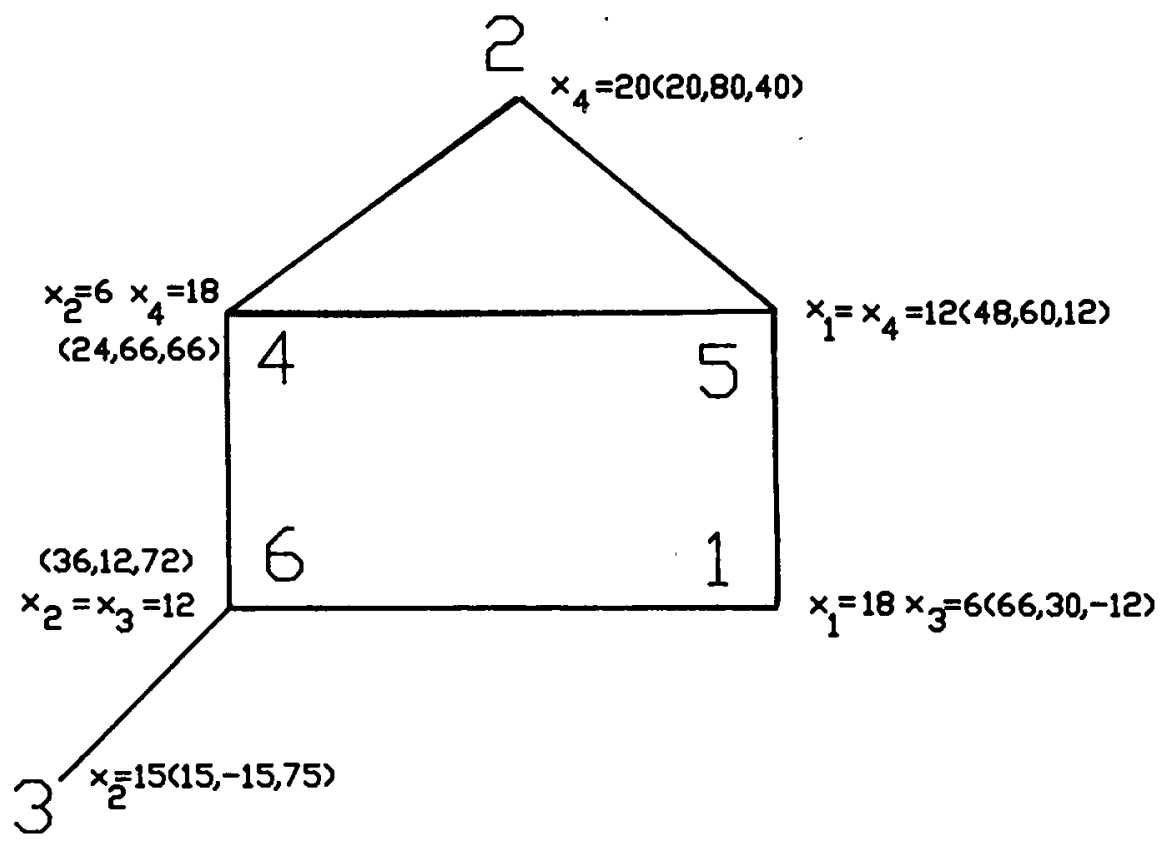

FIGURE 3

A PROJECTION OF THE NONDOMINATED SOLUTIONS 
There is a subfield of management, known as industrial or labor relations, that specializes in the study of labor-management conflicts. The inability of the parties to reach a satisfactory solution may result in strikes, lockouts, or other negative activities. Let us see what we can do to try to avoid such negative outcomes. Consider the value of every possible solution to each of the parties.

Consider Figure 4, which gives a point rating scheme to each party for five levels for each of the four issues. (The five levels are the only ones permitted.) This is obviously a grossly simplified version of a real labor-management negotiation problem. However, depending on the agreement reached (of which the number of possibilities is 625 - four issues each at five levels), we may plot a graph showing the total number of points given to each party for every possible solution. See Figure 5. Just as before we would like to find a nondominated solution. Unfortunately, just as there is no easy way of assuring that the parties to the dispute can resolve their differences, there is no easy way of assuring that we find a nondominated solution in a bargaining situation. If it were possible to draw such a graph for two-party disputes, then such an approach could be used for helping parties to find nondominated solutions.

\begin{tabular}{|c|c|c|c|c|c|}
\hline \multicolumn{3}{|c|}{ WAGE } & \multicolumn{3}{|c|}{ VACATION } \\
\hline Labor & Hage & Mgmt. & Labor & Vac. & Mgmt. \\
\hline Points & Rate (\$) & Points & Points & (Wks.) & Points \\
\hline 129 & 12 & 0 & 75 & 4 & 0 \\
\hline 115 & 10 & 50 & 74 & 3 & 48 \\
\hline 95 & 8 & 90 & 63 & 2 & 83 \\
\hline 50 & 6 & 110 & 38 & 1 & 95 \\
\hline 0 & 4 & 121 & 0 & 0 & 96 \\
\hline \multicolumn{3}{|c|}{ SECURITY } & \multicolumn{3}{|c|}{ EMPLOYEE BENEFIT LEVEL } \\
\hline Labor & Security & Mgmt. & Labor & Benefit & Mgmt. \\
\hline Points & & Points & Points & Level & Points \\
\hline 92 & Vегу High & 0 & 72 & 8 & 0 \\
\hline 87 & High & 25 & 69 & 6 & 45 \\
\hline 70 & Medium & 40 & 53 & 4 & 75 \\
\hline 40 & Low & 53 & 35 & 2 & 89 \\
\hline 0 & None & 63 & 0 & 0 & 91 \\
\hline
\end{tabular}


http://orion.journals.ac.za/

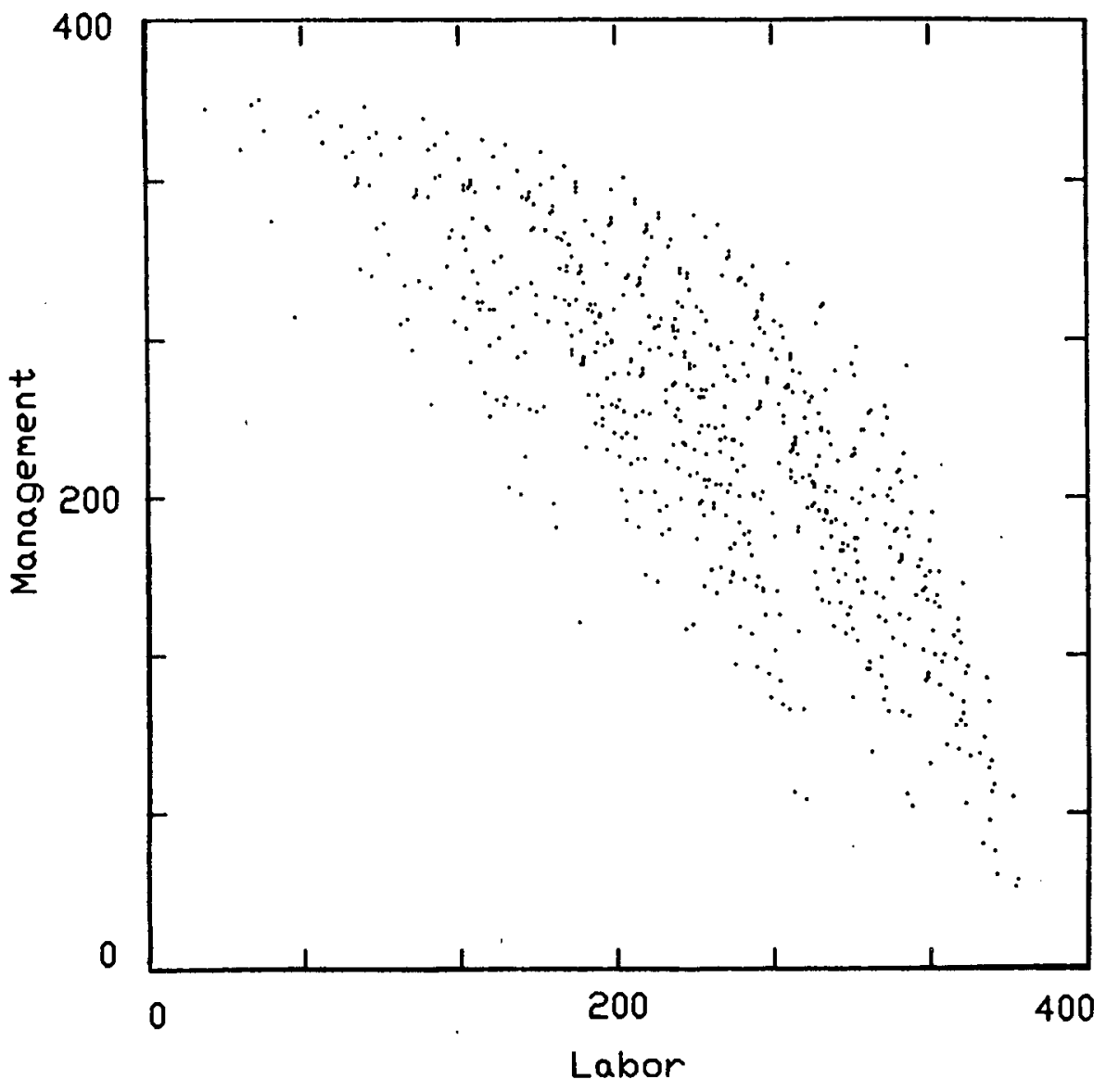

FIGURE 5

A PLOT OF THE 625 SOLUTIONS 


\section{SOME RECENT DEVELOPMENTS IN MCDM}

There are five ideas which have been very popular in recent years in Multiple Criteria Decision Making:

1. Line Search Methods

2. Levels of Aspiration

3. Computer Graphics

4. Cone Dominance

5. The Single Negotiating Text/ Win Win Approach

We shall now consider each of them.

\section{Line Search Methods}

The idea of line search is very old: do a search along a line segment to search for the maximum or the minimum of a function. The approach is a fundamental part of elementary gradient methods in optimization. The method of steepest ascent (descent) is an example of such a method. Assume that we are maximizing an objective function. The idea is that you choose a feasible direction of increase in the function and then move along that direction until a local maximum is achieved. Then we choose a new direction and we search along that direction, and so on. There are problems with the elementary gradient methods in that they zigzag or jam, (i.e., get stuck) resulting in excessive searching with little progress.

Several methods have used line search to advantage. We first discuss the visual interactive method developed by Korhonen and Laakso (1986). It is a microcomputerbased method for solving multiple objective linear programming problems. The method works as follows: (We have taken a bit of poetic license in describing the method.) Without loss of generality, we assume that all objectives are to be maximized.

1. Have the decision maker choose a desired level of each objective that he would like to achieve. This is like goal programming or the related approach of Wierzbicki (1980). Then using a variation of the Wierzbicki approach, project this solution onto the efficient frontier (fiud the "nearest" nondominated solution), and designate the efficient solution as the incumbent solution.

2. Present the incumbent solution to the decision maker, and ask him to specify a new set of desired levels of each objective function that he would now like to achieve. Call this the desired solution, and construct a vector from the incumbent solution to the desired solution (in objective function space). Let that vector have infinite length, thereby extending through and beyond the desired solution. 
3. Analogous to step one, project the vector constructed in step two onto the efficient frontier. The projection constitutes a piecewise-linear function along the efficient frontier. In terms of each objective, as we move along the projection, accordingly, the objectives change in a piecewise-linear manner. Use simple computer graphics to show the changes that occur. See Figure 6.

4. Have the user do a line search (using the computer) along the projection to find his most preferred solution along the projection. As the user moves the cursor along the piecewiselinear segments, the screen displays the values for all of the objective functions. Designate the solution found as the incumbent solution, and go to step 2 . If the incumbent solution remains the same, stop. An optimal solution has been found.

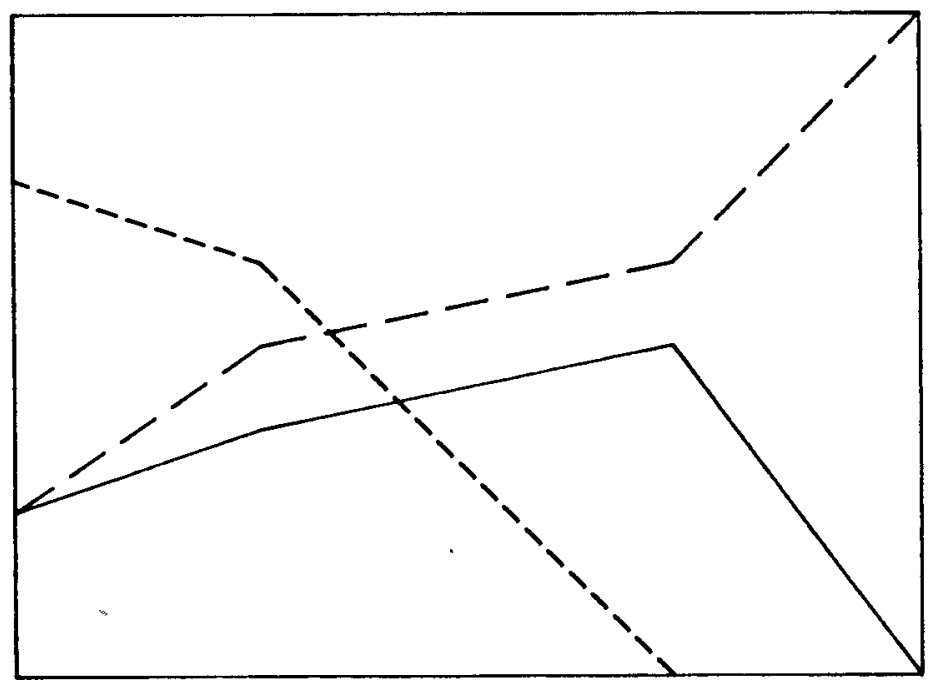

FIGURE 6

AN TXAMPLE OF THE DISPIAY IN THE VISUAL IN'TERACTIVE METHOD 
Refer to the multiple objective linear programming problem discussed earlier. This method will choose the closest nondominated solution point to each of the user's desired solutions. Then the method will connect successive solution points with nondominated solution paths. The latter are the lines from which the user chooses his most preferred solution, before choosing a new desired solution. Though the procedure could conceivably be subject to zigzagging and jamming, that has not been a problem in the authors' experience to date.

The computer implementation is on an 1BM-PC and compatibles and involves the representation of the objectives as piecewise continuous straight line functions. A different color is used for each objective. Some experimentation with the method is described by the authors, and the empirical results with the method to date appear to be reasonable. The method is particularly attractive because of its implementation on a popular microcomputer, the IBM PC.

A second approach that uses the line search approach is a sequel paper by Korhonen and Wallenius (1988). It is called "A Pareto Race" and is almost a video game. (At the Eighth International MCDM Conference held at the Manchester Business School in the U. K. during August, I988, the developers offerred a free copy of their software to the person who could solve a particular problem in the minimum time. A spirited contest ensued. Two people were tied for the minimum, which appeared to be the absolute minimum possible, and two prizes were awarded.) The Pareto Race combines the above approach with the idea of exploring all nondominated solutions, in the sense that the method explores a subset of nondominated solutions, as directed by the user. It may be used to solve a linear programming problem and involves having the decision maker, in a rough sense, explore the efficient frontier by "driving" around on it, thus the similarity to a video game. The Pareto race uses bar graphs to represent the value of each objective. As the user moves around on the efficient frontier, the bar graphs (in color) change.

In the case of two objectives, the corresponding efficient frontier may be thought of as one dimensional, in the case of three objectives, the corresponding efficient frontier may be thought of as two dimensional, for four objectives, the efficient frontier may be thought of as three dimensional, and so on. (The efficient frontier has a useful projection that is one dimension less than the number of objectives.) Accordingly, except for two or three objective problems, it is not possible to represent the efficient frontier graphically.

Using the analogy of "driving" around the frontier, the approach has certain functions that perform the movement, along the frontier. These functions provide certain controls for the user (we take liberties in describing these functions; the authors 
describe them somewhat differently): (Again assume, without loss of generality, that all of the objectives are to be maximized.)

1. Provide movement in a preset direction. This corresponds to a unit movement along a projection described in the visual interactive method.

2. Increase or decrease the speed. This involves changing the stepsize of a unit movement in step one.

3. Change the minimum level of an objective. The minimum level of an objective may be set to a given value, or allowed to be free.

4. Change the direction of the path along the efficient frontier. Increase the component of an objective along the step attempted.

As with the visual interactive method, the Pareto Race has been implemented on an IBM PC or compatible microcomputer. It is easy to use, and has been well received. The authors provide an illustration to a problem and several other applications.

Although the idea of the method and the way in which it is possible to move around the efficient frontier are interesting and worthwhile, the value of the method is greatly enhanced by the computer implementation and the graphics used.

\section{Levels of Aspiration}

Herbert Simon, (see, for example, Simon, 1958 and March and Simon, 1958), has proposed the concept of level of aspiration together with the related concept of satisficing. In solving practical problems, most people choose levels of aspiration. When they find a solution satisfying all levels of (or the level of) aspiration, they accept the solution; otherwise they continue their search or adjust the levels of aspiration. The search and aspiration adjustment process continues until a solution is found. Goal Programming, pioneered by Charnes and Cooper (1961, 1977), and further developed by several researchers, is an example of a multiple objective linear programming method that uses the concept of level of aspiration. In goal programming the user chooses a level of aspiration for each objective and then uses linear programming to find the nearest (using a linear objective function) feasible solution to the chosen levels.

The authors together with a colleague, (Lotfi, Stewart, and Zionts 1988), have developed an approach that uses the idea of aspiration levels with discrete alternative multiple criteria problems. The user wishes to choose one alternative from a number of alternatives, each of which is described in terms of a number of criteria. Objectives may be maximized, minimized, or targeted at a particular range of values (possibly subject to threshold levels), all specified by the user. The user chooses levels of aspiration for each of the criteria, and then adjusts them. As he adjusts the level of 
aspiration for an objective he obtains feedback: the percentage of alternatives that satisfy that level individually and all levels collectively; the "nearest" nondominated feasible solution, plus rankings of all the solutions, and other uselul output. By adjusting his levels of aspiration and obtaining feedback, he can explore nondominated solutions from which to make a choice.

The procedure is embodied in a computer program for the IBM PC or compatible which can handle up to 150 alternatives involving up to ten criteria. A copy of the computer program is available from the authors for a nominal charge.

\section{Computer Graphics}

The use of computer graphics has matured in recent years. As graphics evolved, they have progressed from an esoteric (and expensive) frill to a reasonably-priced scientific tool. The tool allows a vivid visual representation for understanding and design. The colors involved are particularly useful. The development of computer-assisted design and manufacturing has revolutionized production, for example. Though a bit of trite, one picture is worth a 1000 (or more!) words.

In addition to the graphical representations that are being used, there are approaches such as the Chernoff faces (Chernoff, 1973) in which an attribute of a face is indicative of an attribute of a problem. Normal looking faces correspond to normal situations, and peculiar-looking faces correspond to unusual or problem situations. By using faces in this way, it is possible to abstract a multidimensional situation into a picture. We're just beginning to scratch the surface in terms of what we can do using graphics. See also, Korhonnen (1988) for a related application.

The approaches, discussed with respect to line search as well as some of the other methods, utilize computer graphics to a reasonable extent. One line search method shows the objectives as bar graphs, whose lengths change as the objectives change. Another shows a two dimensional line graph with all objectives superimposed as the search proceeds. Some programs include color graphs.

Though one can easily dismiss the color graphics and the graphics as a whole as icing on the cake and nothing more, the proof of the pudding is in the eating! With the color graphics, the programs can be made friendly, easy to use and pleasant to look at as well as interpret. For example, a decision maker observing his weight structure as a bar graph may appreciate the relative significance of higher/lower weights more fully than otherwise. In short, we believe that computer graphics are playing and will continue to play a major role in multiple criteria decision making.

\section{Cone Dominance}

Above we discussed how alternatives that are dominated may be excluded from further consideration so long as we have included all of the criteria in the analysis, and 
so long as all of the criteria fully reflect the problem. We assume that the value function (that we wish to maximize) is an increasing function of all objectives (also to be maximized - no restriction in practice) and is quasi-concave (loosely speaking, it has decreasing returns to scale).

Suppose that one solution, solution one, is preferred to another, solution $\mathbf{k}$. Then we may construct a cone consisting of the halfline from solution one through solution $k$. Any solution that is on the halfline or dominated by the halfline emanating from solution $k$ away from solution one is said to be cone dominated, and therefore less preferred than solution k. In Figure $7 a$ we have illustrated the situation just stated above, and in Figure $7 \mathrm{~b}$, a more general situation in which solutions one, two, and three are all preferred to solution $k$. In the latter case the cone consists of the shaded region. (See Korhonen, Wallenius, and Zionts, 1984, for more information).
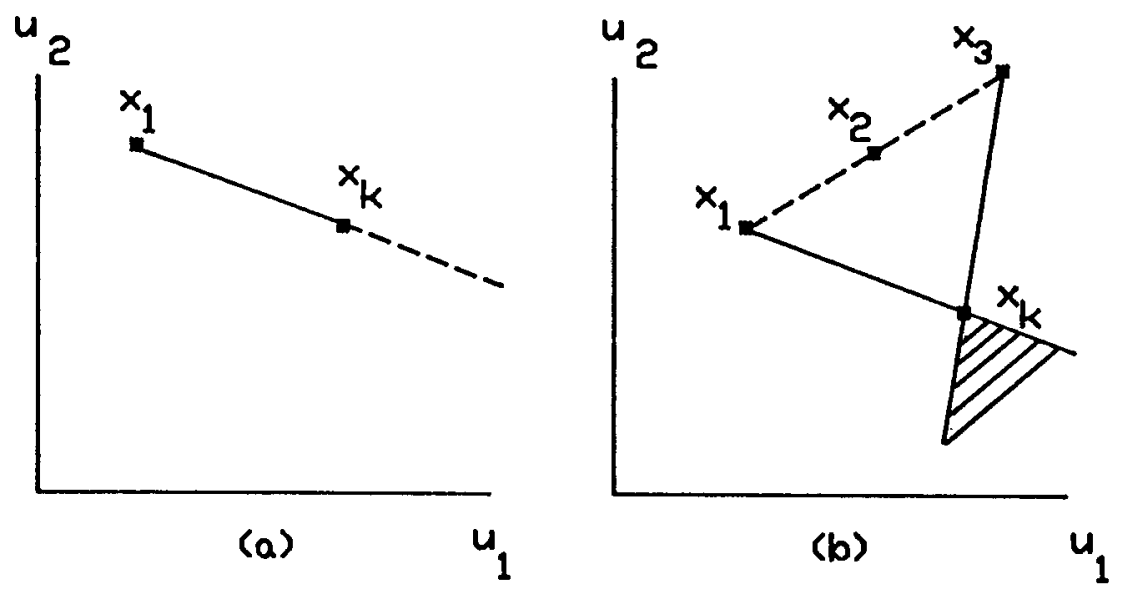

FIGURE 7

\section{SOME HLUSTRATIONS OF CONE DOMINANCE}

The concept of cone dominance is useful because it allows us to eliminate from consideration any solutions that are cone dominated. (We should use the same caveats that we used with respect to ordinary dominance). Eliminating such solutions from further consideration allows us to decrease the number of solutions available and focus 
in on the most preferred ones. Once we have found the most preferred solution or a sufficiently small number of such solutions, we can then rank all of them using some appropriate measure of distance from the most preferred one. In that way we may use secondary considerations in making our final choice. To illustrate the power of cone dominance consider Figure 2. If we knew that alternative 7 were preferred to alternative 10 for that example, then as a result of cone dominance, we could conclude that alternative 10 is preferred to alternatives 11 through 18 . Cone dominance is powerful for problems having any number of objectives, but it is particularly strong for two-objective problems.

Cone dominance is relevant for all of the problems that we have considered. Its use in discrete problems is more convenient because we can eliminate solutions from consideration. For multiple objective linear programming problems, if we eliminate any solutions, then the set of solutions, which was originally convex, becomes nonconvex. Unfortunately there is no simple convenient way to represent a nonconvex set - algebraically. Consequently solutions as encountered must be tested with respect to a cone for cone dominance. They may be eliminated at that time.

\section{The Single Negotiating Text/Win Win Approach}

The Single Negotiating Text/Win-Win Approach is only of use in the group decision problem. Given a group decision problem, the idea is to begin with a solution that is sufficiently poor for all parties that no one likes it. Then the parties search for joint gains whereby they all are happy with the change from the first solution. The procedure continues until there are no further joint gains to be gleaned. One of the proponents of this approach is Raiffa (1982), who describes its use in several high stakes real applications in international relations.

We may explore this idea by considering the problem that we considered in Figure 5. The single negotiating text would be one of the most "southwest" (or dominated) solutions. For example, for the bargaining problem described above, consider a solution having a wage of $\$ 12$ per hour, no vacation, very high security, and a zero level of employee benefits. Such a solution would yield 221 points for labor and 187 points for management. That solution is dominated by many solutions. Beginning with such a solution, the parties would together try to find solutions yielding joint gains, or solutions to the northeast of their starting point. (For example, both labor and management may be willing to trade a lowering of the wage from $\$ 12$ to $\$ 10$ per hour in return for one week's vacation.) In the process both parties would increase their point scores. The procedure continues until there are no solutions to the northeast of the current solution. 


\section{MCDM AND THE ANALYTIC HIERARCHY PROCEDURE}

The Analytic Hierarchy Procedure (AHP) is very popular in South Africa and elsewhere. What is its role vis a vis MCDM? AHP was developed by Prof. Thomas Saaty of the University of Pittsburgh to solve the discrete alternative MCDM problem. (See Saaty 1980 and Saaty and Vargas, 1982.)

An IBM PC implementation of AHP called Expert Choice (EC) is marketed by Decision Support Software, Inc. EC is designed to solve decision analysis problems that can be structured in a hierarchical form. The decision problem is conceptualized as a tree with the root representing an overall goal. Lower level goals are criteria and subcriteria that comprise the overall goal. In Ec, up to seven nodes may be defined for up to seven levels. That is, each goal may consist of up to seven criteria and each criterion in turn may have up to seven subcriteria, and so on. For a problem with up to seven alternatives, the lowest level of the tree represents the alternatives. For a decision problem with more than seven alternatives, the lowest level of the tree represents scales by which the alternatives are rated (using a separate "rating module"). EC can solve problems having as many as 49 objectives and 125 alternatives.

The solution method of AHP is based on pairwise comparisons of criteria and subcriteria to determine the relative importance of criteria. For a large problem having more than seven alternatives, pairwise comparisons are also required to calibrate the rating scales. Consequently, a large number of pairwise comparisons may be necessary. For example, a problem having seven criteria and seven subcriteria (for each criterion) would require 168 pairwise comparisons to determine the weights untilized to rank alternatives. Additional comparisons relating the alternatives and criteria are required. For seven alternatives, for example, the decision maker would have to make an additional 343 pairwise comparisons.

AHP utilizes a measurement scale ranging from $1 / 9$ (absolutely unimportant) through 1 (of equal importance) to 9 (absolutely important) to determine the relative importance of criteria and subcriteria. This scale and its interpretation has been controversial. A criticism of the scale results from possible rank reversal, which means that by adding a duplicate of an alternative to an existing set of alternatives, the ranks that are obtained for a given pair of alternatives may be reversed. (See, for example, Belton and Gear, 1983.) Saaty, Vargas, and Wendell (1983) provide a precise meaning that the importance rating of criteria must have in order to be correct (i.e., not result in rank reversal), The modification of AHP building upon this development is called "Referenced AHP". In a recent article, Schoner and Wedley (1989) show that the referenced AHP requires specific and different interpretations of the meaning of the relative importance of criteria. They argue that conventional AHP (though not the 
referenced AHP) generally yields incorrect answers and potential rank reversal. AHP remains popular, but controversial. It remains to be seen whether its improvements and/or extensions will provide a more robust MCDM method.

Lofti and Teich (1989) explore eight different PC-based MCDM approaches. They discuss the types of problems that can be solved using the different methods and compare the capabilities of the methods and the related computer programs. A list of software studied, and their characteristics is shown in Tables 1 and 2, with permission of the authors.

\section{CONCLUSIONS}

The purpose of this paper was to consider multiple criteria decision making problems and explore some of the recent developments in tbat field. The recent developments we have reviewed are not all so recent (e.g., the ideas of line search and levels of aspiration). In our experience, we have found that ideas that have been around for a while in one area sometimes turn out to be of great value later in that area or another area. This is true with both of the above developments. The ideas themselves are far from new. What is new is their deployment.

Some of the recent developments in multiple criteria decision making are truly exciting. They promise fertile research and application opportunities for the future. The group decision problem remains a particularly difficult and important problem, and considerable research into decision support approaches for attacking that problem is underway. On the other hand, the practical applications of some of the ideas developed leave something to be desired. Though there have been and continue to be situations in which multiple criteria methods have been fruitfully utilized, there is no great abundance of success stories in applying multiple criteria decision models.

What we would like to see in the way of a decision aid for solving multiple criteria problems is an approach that is analogous to the microcomputer electronic spreadsheet, in that it would be a convenient tool that may be easily employed in many decision situations. Our Aspiration Interactive Method, briefly mentioned above, is intended to be a development in that direction. 
Table 1 - Developers and Prices

\begin{tabular}{|c|c|c|}
\hline Software & Source & Cost \\
\hline AIM & $\begin{array}{l}\text { Lotfi, Stewart, and Zionts } \\
\text { Jacobs Management Center } \\
\text { SUNY at Buffalo } \\
\text { Buffalo, NY } 14260\end{array}$ & $\$ 15$ \\
\hline Ariadne & $\begin{array}{l}\text { Ambrose Goicoechea } \\
\text { IS \& Sys. Engineering Dept. } \\
\text { School of Info. and Technology } \\
\text { George Mason University } \\
\text { Fairfax, Virginia }\end{array}$ & $\$ 40$ \\
\hline $\begin{array}{l}\text { Expert } \\
\text { Choice }\end{array}$ & $\begin{array}{l}\text { Decision Support Software, Inc. } \\
1300 \text { Vincent Place } \\
\text { McLean, Virginia } 22101\end{array}$ & $\$ 495^{\star}$ \\
\hline MATS & $\begin{array}{l}\text { Bureau of Reclamation } \\
\text { US Department of Interior } \\
\text { Denver, Colorado } 80225\end{array}$ & Free \\
\hline PCPDA & $\begin{array}{l}\text { Kirkwood and van der Feltz } \\
\text { Dept. of Decision and Info. Sys. } \\
\text { College of Business } \\
\text { Arizona State University } \\
\text { Tempe, Arizona } 85287\end{array}$ & $\$ 20$ \\
\hline$P / G \%$ & $\begin{array}{l}\text { Decision Aids, Inc. } \\
361 \text { Lincoln Hall } \\
\text { University of } 111 \text { inois } \\
\text { Urbana, Ill } 61801\end{array}$ & $\$ 20$ \\
\hline VIMDA & $\begin{array}{l}\text { NumPl an } \\
\text { P.0. Box } 22 \\
00421 \text { Helsinki } \\
\text { Finland }\end{array}$ & $\$ ?$ \\
\hline VIG & NumPlan & $\$ 4000$ \\
\hline
\end{tabular}

? unknown at the time of this report

- list price, educational institutions may qualify for a discount

c academic price, price for industry is $\$ 1,900$. 
Table 2 - Software Characteristics*

\begin{tabular}{|c|c|c|c|}
\hline Software & $\begin{array}{l}\text { Problem } \\
\text { Type }\end{array}$ & $\begin{array}{l}\text { System } \\
\text { Required }\end{array}$ & $\begin{array}{l}\text { Problem } \\
\text { Size }\end{array}$ \\
\hline AlM & $\begin{array}{l}\text { Discrete } \\
\text { Alternative }\end{array}$ & $\begin{array}{l}R=256 \\
D=C\end{array}$ & $\begin{array}{l}10 \text { obj. } \\
150 \text { alt. }\end{array}$ \\
\hline Ariadne & $\begin{array}{l}\text { Discrete } \\
\text { Alternative }\end{array}$ & $\begin{array}{l}R=256 \\
D=G / C\end{array}$ & $\begin{array}{l}15 \text { att. } \\
10 \text { alt. }\end{array}$ \\
\hline $\begin{array}{l}\text { Expert } \\
\text { Choice }\end{array}$ & $\begin{array}{l}\text { Discrete } \\
\text { Alternative }\end{array}$ & $\begin{array}{l}R=320 \\
D=M / G / C\end{array}$ & $\begin{array}{l}49 \text { obj. } \\
125 \text { alt. }\end{array}$ \\
\hline MATS & $\begin{array}{l}\text { Discrete } \\
\text { Alternative }\end{array}$ & $\begin{array}{l}R=256 \\
D=M / G / C\end{array}$ & $\begin{array}{l}40 \text { obj. } \\
40 \text { alt. }\end{array}$ \\
\hline PCPDA & $\begin{array}{l}\text { Stochastic } \\
\text { MAUF }\end{array}$ & $\begin{array}{l}R=76 \\
D=M\end{array}$ & $\begin{array}{ll}20 & \text { obj. } \\
20 & \text { alt. }\end{array}$ \\
\hline$P / G \%$ & $\begin{array}{l}\text { Discrete } \\
\text { Al ternative }\end{array}$ & $\begin{array}{l}R=256 \\
D=M\end{array}$ & $\begin{array}{l}15 \text { obj. } \\
15 \text { alt. }\end{array}$ \\
\hline VIMDA & $\begin{array}{l}\text { Discrete } \\
\text { Alternative }\end{array}$ & $\begin{array}{l}R=256 \\
D=G / C\end{array}$ & $\begin{array}{ll}10 & \text { obj } \\
500 & \text { alt. }\end{array}$ \\
\hline VIG & MOLP & $\begin{array}{l}R=256 \\
D=G / C\end{array}$ & $\begin{array}{l}10 \text { obj. } \\
100 \text { rows } \\
96 \text { var. }\end{array}$ \\
\hline
\end{tabular}

* All of the packages could be used on a one floppy drive system, availability of a hard drive enhanced their performance.

Legend $\quad R=$ random access memory in kilo-bytes

$D=$ type of display: $M$ - monochrome, $G$ - graphics, $C$ - color 


\section{REFERENCES}

1. Belton, V. and A.E. Gear, "On a Shortcoming of Saaty's method of analytic hierarchies", Omega, 11, 3, 1983, 228-230.

2. Charnes, A. and W.W. Cooper, Management Models and Industrial Applications of Linear Programming, John Wiley and Sons, New York, 1961.

3. Charnes, A. and W.W. Cooper, "Goal Programming and Multiple Objective Optimization - Part 1," Europenn Journal of Operations Research, I, 1977, 39.

4. Chernoff, H. "Using Faces to Represent Points in k-Dimensional Space Graphically," Journal of the American Statistical Associalion, 68, 1973, 361-368.

5. Climaco, J.C.N. and C.H. Antunes, "Trimap: An Interactive Tricriteria Linear Programming Package," Foundations of Control Engineering, 12, 1987.

6. Korhonen, P., "Using Harmonious Houses for Visual Pairwise Comparison of Multiple Criteria Alternatives", Working Paper F-203, Helsinki School of Economics, Helsinki, Finland (1988).

7. Korhonen, P.J. and J. Laakso, "A Visual Interactive Method for Solving the Multiple Criteria Problem," European Journal of Operational Research, 24, 1986, 277-287.

8. Korhonen, P., J. Wallenius, and S. Zionts, "Solving the Discrete Multiple Criteria Problem Using Convex Cones," Management Science, 30, 11, 1984, 1336-1345.

9. Korhonen, P. and J. Wallenius, "A Pareto Race," Noval Research Logistics, 35, (6), 1988, 615-623.

10. Lotfi, V., T.J. Stewart, and S. Zionts, "An Aspiration-Level Interactive Model for Multiple Criteria Decision Making," Working Paper No. 711, School of Management, State University of New York at Buffalo, 1988.

11. Lotfi, V. and J.E. Teich, "On Overview of Recent PC-Based Multiple Criteria Decision Making (MCDM) Software", Working Paper No. 727, School of Management, State University of New York at Buffalo, 1989.

12. March, J. and H.A. Simon, Organizations, John Wiley, New York, 1958.

13. Raiffa, H., The Art and Science of Negotiation, Belknap Press of Harvard University Press, Cambridge, Mass., 1982.

14. Simon, H.A., Administrative Behavior, MacMillan, New York, 1958.

15. Saaty, T.L., The Analytic Hierarchy Process, McGraw Hill, New York, 1980.

16. Saaty, T.L. and L. Vargas, The Logic of Priorities, Kluwer-Nijhoff, Boston, 1982.

17. Saaty, T.L., L.G. Vargas and R.E. Wendell, "Assessing Attribute Weights by Ratios", Omega, 11, 1, 9-12, 1983.

18. Schoner, B. and W.C. Wedley, "Ambiguous Criteria Weight in AHP: Consequences and Solution", Decision Sciences, 20, 3, 1989, 462-475. 
http://orion.journals.ac.za/

19. Steuer, R.E., Multiple Criteria Optimization Theory, Computation, and Application, John Wiley and Sons, New York, 1986.

20. Wierzbicki, A.P., "The Use of \#eference Objectives in Multi-objective Optimization," G. and T. Gal (Eds.) Multiple Criteria Decision Making Theory and Application, Springer-Verlag, New York, 1980. 